\title{
Reconstruction of Continuous and Focalized Brain Functional Source Images From Electroencephalography
}

\author{
Chang-Hwan Im ${ }^{1}$, Hyun-Kyo Jung ${ }^{2}$, Ki-Young Jung ${ }^{3}$, and Soo Yeol Lee ${ }^{4}$ \\ ${ }^{1}$ Department of Biomedical Engineering, Yonsei University 234 Maeji, Heungeop, Wonju, 220-710 Korea \\ ${ }^{2}$ School of Electrical Engineering, Seoul National University, Seoul, 151-744 Korea \\ ${ }^{3}$ Department of Neurology, College of Medicine, Korea University, Seoul, 136-701 Korea \\ ${ }^{4}$ Department of Biomedical Engineering, Kyung Hee University, Yongin, 446-701 Korea
}

In this paper, a new hybrid approach to reconstruct more accurate brain functional source images from electroencephalography is proposed. The proposed approach combines extended source model and focal underdetermined system solution algorithm. Feasibility studies with realistic simulation data and the epilepsy patient's data demonstrate that continuous, as well as focalized, brain electrical source images can be reconstructed utilizing the proposed approach.

Index Terms-Brain functional source image, cortical source imaging, electroencephalography (EEG), focal underdetermined system solution (FOCUSS), linear inverse problem.

\section{INTRODUCTION}

$\mathbf{E}$ LECTROENCEPHALOGRAPHY (EEG) source imaging is a kind of electromagnetic inverse problems which inversely estimates neuronal electrical source distribution on human brain cortex from noninvasive measurements of electric potentials at multichannel scalp electrodes [1]. Since most EEG generators (neuronal sources) are confined within very thin ( 4 mm) gray matter of the cerebral cortex, of which the primary current directions are oriented perpendicularly to the outmost surface, recent studies have restricted possible source space only on the cortical surface. The source intensities were then estimated using various inverse algorithms [1]-[5].

The reconstructed sources, however, are usually very widespread and discontinuous due to various external disturbances, brain noises, ill-posed characteristics of the inverse operator, lack of regularization schemes, etc. [4], which makes it difficult for us to estimate true source locations precisely. To solve those problems, two antithetical types of methods have been used: smoothing-type methods such as low resolution elecmagnetic tomography (LORETA) [6] and extended source model [7]; focusing-type methods such as $\mathrm{L} p$ norm estimation $(1 \leq p<$ 2 ) [8] and focal underdetermined system solution (FOCUSS) [9]. The source distribution by the smoothing-type methods is very continuous, but often too widespread, while that by the focusing-type methods is too focalized or discontinuous to describe actual source extension.

Some studies have tried to combine the two different kinds of methods. The self-coherence enhancement algorithm (SCEA)

Digital Object Identifier 10.1109/TMAG.2006.892282

Color versions of one or more of the figures in this paper are available online at http://ieeexplore.ieee.org.
[10] and the shrinking LORETA/FOCUSS [11], [12] are two examples which tried to combine LORETA and FOCUSS. Due to the intrinsic characteristic of LORETA which requires spatial Laplacian in the regularization operator, however, those algorithms are very difficult to be applied to the cortical source imaging which uses irregular cortical surface elements. Another approach was to combine extended source model with L $p$ norm estimation [7]. Although the extended source model was readily applicable to the highly folded cortical surface sources, the hybrid approach had two major problems: 1) the L $p$ norm estimation requires heavy computational cost because of the nonlinear optimization using linear program-ing algorithms; 2) the focalization level cannot be readily controlled without changing $p$. Please note that the change of $p$ value also requires another time-consuming nonlinear iteration.

In this paper, we combined the extended source model and FOCUSS to reconstruct continuous as well as focalized brain source images in a more efficient manner. Any inexperienced users can readily control the smoothness and focalization level in the hybrid approach and then quickly and precisely obtain brain source images which describe actual source distribution. This approach was applied to realistic EEG simulations and epilepsy patient's data and compared with conventional ones.

\section{METHODS}

\section{A. Anatomical and Physiological Constraints for EEG Cortical Source Imaging}

As briefly introduced before, main EEG generators are oriented in parallel, perpendicular to the cortical surface. Nowadays, this physiological phenomenon has been adopted successfully and used as a basic anatomical constraint in EEG source imaging [1]-[5]. The source imaging with the anatomical constraint resulted in elimination of spurious sources [2], as well 
as reduction of crosstalk distribution [3], compared to conventional voxel (volume pixel) based imaging techniques.

To impose the anatomical constraint, many dipolar sources are placed on cortical surface, usually on the interface between white and gray matter of the cerebral cortex extracted from structural MRI, which is relatively easier to be detected than the other borders [13]. We can further constrain each of these dipolar sources to be normal to the surface. Then, the strengths of the dipolar sources are determined using linear $\left(\mathrm{L}_{2}\right.$ norm) or nonlinear (Lp norm) estimation methods [1], [4]. To determine proper locations and orientations of the scattered sources, the cortical surface is usually tessellated into huge number of triangular elements, the number of which often exceeds several hundreds of thousands. Developments of medical image processing techniques and high resolution structural MRI enabled us to get high resolution cortical surface with submillimeter modeling errors [13]. Unfortunately, however, it is computationally inefficient to use whole cortical surface vertices for the source reconstruction considering intrinsic spatial resolution of EEG. To reduce the number of possible source locations, small number of vertices is downsampled from the cortical surface as regularly as possible and used for source reconstruction purpose, whereas the original mesh information is used only for visualization purpose [14].

\section{B. Conventional Linear Inverse Estimation}

The variable of the conventional source model is the current intensity of each dipolar source. Then, the relation between the current intensity and the measured data can be expressed as

$$
\mathbf{x}=\mathbf{L I}+\mathbf{n}
$$

where $\mathrm{x}$ is a column vector gathering the measurements on $N_{M}$ sensors at a given time instant; $\mathbf{I}$ is a column vector made of the $N$ corresponding current source intensities; $\mathbf{L}$ is the $N_{M} \times N$ lead field matrix; $\mathbf{n}$ is a perturbation or noise vector. The lead field $A_{j i}$ is defined as electromagnetic quantity of $j$ th sensor induced by $i$ th dipolar source with unit intensity. In the present study, boundary element method (BEM) considering realistic geometry head model [15] was applied for forward calculation.

We used a linear estimation approach [4] to reconstruct cortically distributed brain sources. The expression for the inverse operator $\mathbf{W}$ is

$$
\mathbf{W}=\mathbf{R L}^{\mathrm{T}}\left(\mathbf{L R L}^{\mathrm{T}}+\lambda^{2} \mathbf{C}\right)^{-1}
$$

where $\mathbf{R}$ is a source covariance matrix and $\mathbf{C}$ is a noise covariance matrix. The source distribution can be estimated by multiplying the measured signal at a specific instant $\mathbf{x}$ by $\mathbf{W}$. In this work, the source covariance matrix $\mathbf{R}$ was assumed to be a diagonal matrix, which means that we ignored relationships between neighboring sources. Prestimulus time window was used to calculate C. $\lambda^{2}$ is a regularization parameter and was determined using an L-curve method [16].

\section{Extended Source Model and FOCUSS}

Extended source model assumes spatially distributed functions centered at every source location and then estimates their coefficients [9]. In this work, we used a Gaussian function defined at a source location $i$ as

$$
f_{i}(x)=A_{i} \exp \left(-d_{x i, x}^{2} / 2 \sigma^{2}\right)
$$

where $f_{i}(x)$ is the functional value at an arbitrary point $x, d_{x i, x}$ is the Euclidean distance between $i$ th source location $x_{i}$ and $x$, $A_{i}$ is the maximum of the $i$ th source, and $\sigma$ is a predetermined constant which controls extension of the source. The Gaussian source approximation is physiologically plausible because the actual brain source activation is decaying from a certain center position and smoothly diminished at the border [7], [17]. Then, current source intensity $I_{j}$ at $j$ th source location $x_{j}$ can be evaluated by summing the $N$ functional values at $x_{j}$ as

$$
I_{j}=\sum_{(i=1, N)} f_{i}\left(x_{j}\right)=\sum_{(i=1, N)} A_{i} \exp \left(-d_{i, x j}^{2} / 2 \sigma^{2}\right) .
$$

Equation (4) can be rewritten as a matrix form $\mathbf{I}=\mathbf{E A}$, where $\mathbf{A}$ is a vector consisting of coefficients $A_{i}(i=1, \ldots, N)$. The relationship between measured electrical potential vector $\mathbf{x}$ and the current source intensity vector $\mathbf{I}$ then becomes

$$
\mathbf{x}=\mathbf{L I}+\mathbf{n}=\mathbf{L E A}+\mathbf{n}=\mathbf{T A}+\mathbf{n}
$$

The vector $\mathbf{A}$ is estimated by applying the linear inverse operator $\mathbf{A}=\mathbf{R} \mathbf{T}^{\prime}\left(\mathbf{T R T}^{\prime}+\lambda^{2} \mathbf{C}\right)^{-1} \mathbf{I}$, where the processes are the same as the conventional approach in (2) except for $\mathbf{T}$ matrix.

FOCUSS [9] is a kind of recursive weighting algorithms which imposes 'probability to be estimated' on each source according to previous current intensity values. We reflected the values to the diagonal terms of $\mathbf{R}$, as follows:

$$
R_{i i}^{(k+1)}=R_{i i}^{(k)}\left(I_{i} / I_{\mathrm{Max}}\right)
$$

where $R_{i i}$ is the $i$ th diagonal term of $\mathbf{R}$, the superscript $(k)$ represents rhw $k$ th iteration, $I_{\mathrm{Max}}$ is the maximum current intensity, and $R_{i i}^{(0)}=1$ for all $i$. Since the FOCUSS iteration requires only inversion of $N_{M} \times N_{M}$ matrix ( $N_{M}$ is usually not more than 128) and a single matrix multiplication process, the increment of computational cost is not significant compared to the L $p$ norm estimation, which requires time-consuming linear programming iteration. Moreover, one can readily control the smoothness and focalization level of the source distributions just by adjusting $\sigma$ in (3) and the number of FOCUSS iteration.

\section{Simulations AND RESUltS}

Neuroelectromagnetic inverse problems (NIP) are hard to be verified by in vivo experiments because exact source locations inside of a real human brain cannot be estimated $a$ priori. Thus, artificial forward data simulated with cortical patches are widely used to validate the NIP solutions [18].

\section{A. Simulation Setups}

We adopted realistic conditions to construct artificial EEG data. We assumed 128 electrodes that were attached on a subject's scalp. To utilize anatomical information, interface between white and gray matter was extracted from MRI T1 images of a standard brain model and tessellated into 865712 

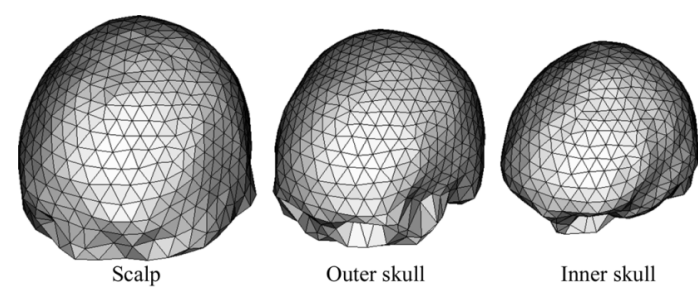

Fig. 1. Boundary element model for EEG forward calculations.
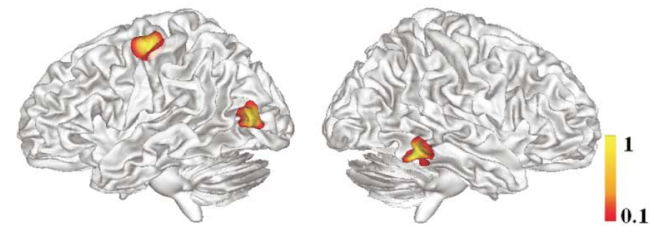

Fig. 2. Example of cortical patch activations: Three simultaneous Gaussian distributed sources were assumed in both hemispheres.

triangular elements and 432654 vertices using BrainSuite software package [19]. For more accurate forward calculations, a three-layer boundary element model (Fig. 1), consisting of inner and outer skull boundaries and scalp surface, was used [15]. 5372 boundary elements and 2748 surface nodes were generated from the same structural MRI data. Cortical patches to construct artificial EEG data were made of a set of dipoles with Gaussian distributed current intensity profile and orientations perpendicular to the tessellated cortical surface. The maximum of the Gaussian function was set as

$$
\begin{aligned}
A & =-0.6 \times 10^{-4}(t-100)^{2}+0.6 \quad(0 \mathrm{~ms} \leq t<200 \mathrm{~ms}) \\
& =0 \quad(200 \mathrm{~ms} \leq t<400 \mathrm{~ms}) .
\end{aligned}
$$

After calculating electrical potentials at the 128 electrodes assuming $200-\mathrm{Hz}$ sampling rate, we added real brain noise, which was obtained from a prestimulus period of a practical EEG experiment. The original signal without noise was scaled in order for SNR to be approximately $7 \mathrm{~dB}$. Fig. 2 shows an example of the assumed cortical patch activations.

\section{B. Intuitive Comparisons}

We compared brain source images obtained from five different cases: (Case I) Conventional linear inverse estimation without smoothing or focusing steps; (Case II) Extended source model with $\sigma=10 \mathrm{~mm}$; (Case III) Extended source model with $\sigma=20 \mathrm{~mm}$; (Case IV) three FOCUSS iteration on Case I result; (Case V) four FOCUSS iteration on Case IV result. Fig. 3 shows the source estimates at $100 \mathrm{~ms}$ applied to an example shown in Fig. 2. It can be seen from the figures that the source distributions obtained from the conventional technique are discontinuous and include many noisy sources. As seen in Fig. 3(b) and (c), larger value of $\sigma$ results in more widespread and smoother source distribution. The application of FOCUSS algorithm to the conventional source model focalized the original source distribution, but the distribution was still discontinuous [see Fig. 3(d)]. On the contrary, we could get focalized as well as continuous source distribution by using the proposed hybrid approach, as presented in Fig. 3(e).

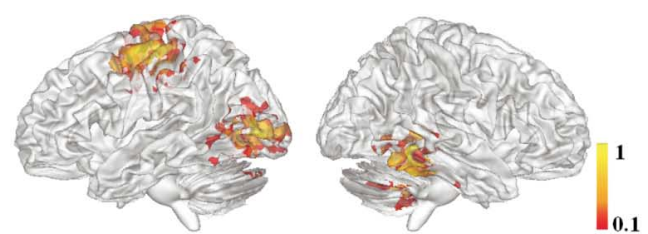

(a)

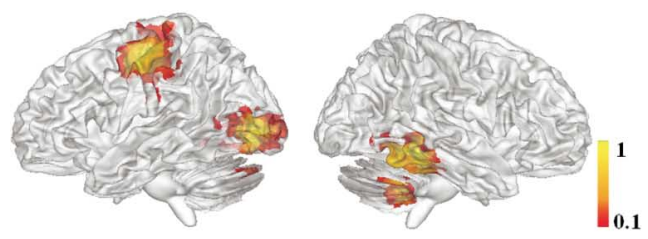

(b)

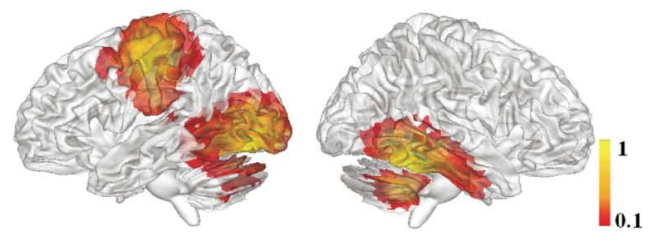

(c)

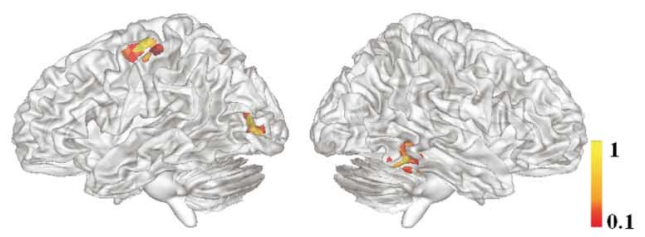

(d)

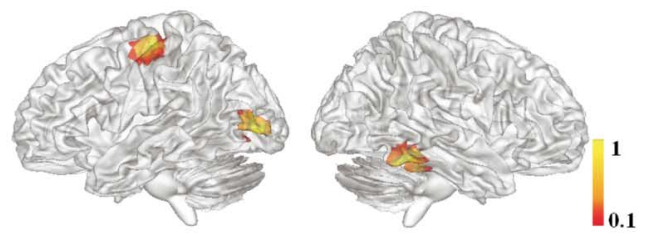

(e)

Fig. 3. Source distributions applied to EEG activations shown in Fig. 2 under different cases. (a) Case I: Conventional MNE. (b) Case II: Extended source, $\sigma=10 \mathrm{~mm}$. (c) Case III: Extended source, $\sigma=20 \mathrm{~mm}$. (d) Case IV: Three FOCUSS iteration in Case I result. (e) Case V: Four FOCUSS iteration in Case III result.

TABLE I

AVERAGEd DF AND NSS VALUES FOR Five CASES (100 Simulations)

\begin{tabular}{cccccc}
\hline Cases & Case I & Case II & Case III & Case IV & Case V \\
\hline DF & 0.085 & 0.073 & 0.031 & 0.543 & 0.514 \\
NSS & 31.3 & 14.4 & 7.0 & 24.7 & 11.4 \\
\hline
\end{tabular}

\section{Quantitative Comparisons}

For more quantitative comparisons, two assessment criteria called degree of focalization (DF) and the number of spurious solutions (NSS) were introduced [20]. DF is defined as source power contained in patch areas exceeding a certain threshold (0.1 here) divided by overall source power. Thus, higher DF implies that the method can reconstruct more focalized source distribution. NSS represents the number of local peaks outside the patch areas, where the local peak is defined as a point which has larger value than any neighboring points. Thus, larger NSS implies that larger numbers of noisy or spurious sources are generated. We generated 100 artificial EEG data sets from different source locations and evaluated the DF and NSS values of Case I-Case V. We can see from the results presented in Table I that 


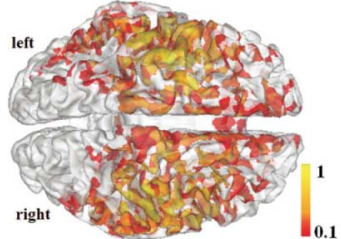

(a)

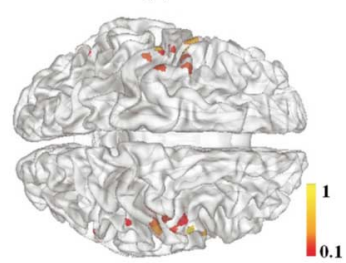

(c)

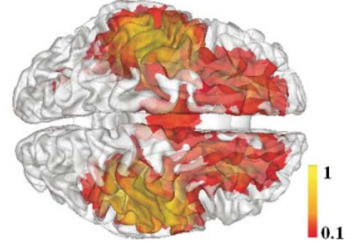

(b)

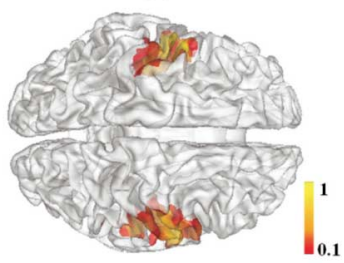

(d)
Fig. 4. Normalized source distribution estimated from various methods: (a) conventional model, (b) extended source $(\sigma=10 \mathrm{~mm})$, (c) FOCUSS (iteration $=3)$, $(\mathrm{d})$ proposed hybrid method $(\sigma=10 \mathrm{~mm}$, and iteration $=3$ ).

1) the use of extended source model (Cases II and III) significantly reduced spurious sources (small NSS), but resulted in too widespread distributions (small DF); 2) the use of FOCUSS algorithm (Case IV) enhanced focal characteristic (large DF), but still showed significant number of spurious sources (large NSS); 3 ) the use of the proposed model (Case V) enhanced both focal characteristic (large DF) and smoothness of the images (small NSS) and yielded most compromising estimates.

\section{PRACTICAL APPLICATION-EPILEPSY DATA}

Benign Rolandic epilepsy of childhood (BREC) is a common primary partial epilepsy syndrome, which usually appears to children under age of 15 [21]. Interictal spike waveform of a child whose spike can be viewed as bilaterally synchronous discharge (BSD) was measured using a 45-channel scalp EEG system. The EEG source distribution was reconstructed at a major spike peak. Fig. 4 shows the epileptic source distribution reconstructed using different techniques. It can be seen from Fig. 4(a) that the conventional inverse estimation resulted in very widespread and discontinuous source images as observed previously in simulation studies. Fig. 4(b)-(d) shows the source estimates obtained from the extended source model $(\sigma=10 \mathrm{~mm})$, FOCUSS (iteration $=3$ ), and the proposed hybrid approach. It can be seen from the results that the extended source model and FOCUSS resulted in too widespread and discontinuous distributions, respectively, to identify exact locations or extensions of actual sources; while the proposed approach could yield better brain source images.

\section{CONCLUSION}

In this study, a new hybrid approach which combines the extended source model and the FOCUSS algorithm to reconstruct brain electrical source images more accurately and efficiently. Quantitative simulation studies and clinical data analysis demonstrated that the proposed approach can be a promising technique to overcome the tradeoff between smoothness and focalization in brain source estimation. This approach can be applied to magnetoencephalography source imaging as well since both MEG and EEG use the same inverse operators.

\section{ACKNOWLEDGMENT}

This work was supported by a Grant from the Korea Health 21 R\&D project, Ministry of Health and Welfare, Korea (02PJ3-PG6-EV07-0002).

\section{REFERENCES}

[1] M. Fuchs, M. Wagner, T. Kohler, and H.-A. Wischmann, "Linear and nonlinear current density reconstructions," J. Clin. Neurophysiol., vol. 16, pp. 267-295, 1999.

[2] S. Baillet, J. J. Riera, G. Marin, J. F. Mangin, J. Aubert, and L. Garnero, "Evaluation of inverse methods and head models for EEG source localization using a human skull phantom," Phys. Med. Biol., vol. 46, pp. 77-96, 2001.

[3] A. K. Liu, J. W. Belliveau, and A. M. Dale, "Spatiotemporal imaging of human brain activity using functional MRI constrained magneto-encephalography data: Monte Carlo simulations," Proc. Natl. Acad. Sci. $U S A$, vol. 95, pp. 8945-8950, 1998.

[4] A. M. Dale and M. I. Sereno, "Improved localization of cortical activity by combining EEG and MEG with MRI surface reconstruction: a linear approach," J. Cogn. Neurosci., vol. 5, pp. 162-176, 1993.

[5] C. H. Im, H. K. Jung, and N. Fujimaki, "fMRI constrained MEG source imaging and consideration of fMRI invisible sources," Hum. Brain Mapp., vol. 26, no. 2, pp. 110-118, 2005.

[6] R. M. Pascual-Marqui, C. Michel, and D. Lehman, "Low resolution electromagnetic tomography: A new method for localizing electrical activity in the brain," Int. J. Psychophysiol., vol. 18, pp. 49-65, 1994.

[7] M. Wagner, T. Köhler, M. Fuchs, and J. Kastner, "An extended source model for current density reconstructions," in Proc. BIOMAG, 2000, pp. 749-752.

[8] J. Yao and J. P. A. Dewald, "Evaluation of different cortical source localization methods using simulated and experimental EEG data," Neuroimage, vol. 25, no. 2, pp. 369-382, 2005.

[9] I. F. Gorodnistky, J. S. George, and B. D. Rao, "Neuromagnetic imaging with FOCUSS: a recursive weighted minimum norm algorithm," Electroencephalogr. Clin. Neurophysiol., vol. 95, pp. 231-251, 1995.

[10] D. Z. Yao and B. He, "A self-coherence enhancement algorithm and its application to enhancing three-dimensional source estimation from EEGs," Ann. Biomed. Eng., vol. 29, no. 11, pp. 1027-1029, 2001.

[11] H. Liu, X. Gao, P. H. Schimpf, F. Yang, and S. Gao, "A recursive algorithm for the three-dimensional imaging of brain electric activity: Shrinking LORETA-FOCUSS," IEEE Trans. Biomed. Eng., vol. 51, no. 10 , pp. $1794-1802$, Oct. 2004

[12] H. Liu, P. H. Schimpf, G. Dong, X. Gao, F. Yang, and S. Gao, "Standardized shrinking LORETA-FOCUSS (SSLOFO): A new algorithm for spatio-temporal EEG source reconstruction," IEEE Trans. Biomed. Eng., vol. 52, no. 10, pp. 1681-1691, Oct. 2005.

[13] B. Fischl and A. M. Dale, "Measuring the thickness of the human cerebral cortex from magnetic resonance images," Proc. Natl. Acad. Sci. USA, vol. 97, pp. 11050-11055, 2000.

[14] C. H. Im, H. K. Jung, and N. Fujimaki, "Anatomically constrained dipole adjustment (ANACONDA) for accurate MEG/EEG focal source localizations," Phys. Med. Biol., vol. 50, pp. 4931-4953, 2005.

[15] M. S. Hämäläinen and J. Sarvas, "Realistic conductivity geometry model of the human head for interpretation of neuromagnetic data," IEEE Trans. Biomed. Eng., vol. 36, no. 2, pp. 165-171, Feb. 1989.

[16] P. Hansen, "Analysis of discrete ill-posed problems by means of the L-curve," SIAM Rev., vol. 34, pp. 561-580, 1992.

[17] E. R. Kendal, J. H. Schwartz, and T. M. Jessell, Principles of Neural Science, 4th ed. New York: McGraw-Hill, 2000.

[18] C. H. Im, C. Lee, H. K. Jung, Y. H. Lee, and S. Kuriki, "Magnetoencephalography cortical source imaging using spherical mapping," IEEE Trans. Magn., vol. 41, no. 5, pp. 1984-1987, Sep. 2005.

[19] D. W. Shattuck and R. M. Leahy, "BrainSuite: An automated cortical surface identification tool," Med. Image Anal., vol. 6, pp. 129-142, 2002.

[20] C. H. Im, K. O. An, H. K. Jung, H. Kwon, and Y. H. Lee, “Assessment criteria for MEG/EEG cortical patch tests," Phys. Med. Biol., vol. 48, pp. 2561-2573, 2003.

[21] Y. Y. Lin, K. P. Chang, J. C. Hsieh, T. C. Yeh, H. Y. Yu, S. Y. Kwan, D. J. Yen, C. H. Yiu, and R. Hari, "Magnetoencephalographic analysis of bilaterally synchronous discharges in benign Rolandic epilepsy of childhood," Seizure, vol. 12, pp. 448-455, 2003

Manuscript received April 20, 2006 (e-mail: ich@yonsei.ac.kr). 